\title{
DESIGN AND EXPERIMENTAL EVALUATION OF QUADRATURE OSCILLATOR EMPLOYING SINGLE FB-VDBA
}

\author{
Abdullah Yesil — Firat Kacar — Koray Gurkan *
}

\begin{abstract}
This paper presents an attractive and a new voltage-mode quadrature oscillator using a single Fully Balanced-Voltage Differencing Buffer Amplifier (FB-VDBA) as the active element. The circuit structure is very simple, consisting of merely one FB-VDBA, one resistor and two capacitors. The circuit is implemented using the commonly available OPA860 which results in low output impedance and high current drive capability. The proposed circuit also has a suitable architecture for IC production. Experimental results which are matched well with the theoretical assumptions are given.
\end{abstract}

K e y w or ds: quadrature oscillator, experimental results, voltage mode, CMOS active elements

\section{INTRODUCTION}

An essential circuit blocks of a typical telecommunications, measurements and signal processing systems are constructed with the quadrature sinusoidal oscillators that generate two sinusoidal outputs which have same frequency but $90^{\circ}$ phase difference [1-3]. At present, there is a great interest in the realization of various analog signal processing circuits using the Fully Balanced Voltage Differencing Buffer Amplifier (FB-VDBA) as an active element [4-8]. The FB-VDBA, particularly the commercially available integrated circuit type OPA860 providing an accessible compensation terminal, is very versatile building block for analog circuit designs because of several advantages. It provides over the voltage-mode operational amplifier (VOA) and ease of designing various functional circuits with the least possible number of external components.

As a result, many quadrature oscillator circuits have been reported in the literature [9-17] using different type of active elements. Designers of quadrature oscillators utilize various active elements such as multifunction current differencing cascaded transconductance amplifier [9], current differencing transconductance amplifier (CDTA) [10], operational amplifier [11], current differencing buffered amplifier (CDBA) [12], current feedback operational amplifier (CFOA) [13], differential difference current conveyor (DDCC) [14], current follower transconductance amplifier (CFTA) [15], fully differential current conveyor (FDCCII) [16], and double current controlled current feedback amplifier (DCC-CFA) [17]. However, the oscillators mentioned above suffer from one or more disadvantages;

- having two or three active elements [10-15,17]

- consisting of high number of passive components $[11-14,16]$

- not being suitable for voltage mode filter structure due to high output impedance $[10,12,14,16]$
The purpose of this paper is to introduce a voltagemode quadrature oscillator, based on a single FB-VDBA. The circuit consists of one FB-VDBA, one resistor, and two capacitors. The circuit is implemented using the commonly available OPA860 which results in low output impedance and high current drive capability. Finally, this oscillator has been built by means of a FB-VDBA which has been realized with OPA860. The experimental results are matched with theoretical analysis.

\section{FULLY BALANCED VOLTAGE DIFFERENCING BUFFER AMPLIFIER}

The schematic symbol of the FB-VDBA is given in Fig. 1. The model can be described by the following set of circuit equations.

$$
\left(\begin{array}{c}
I_{\mathrm{v}+} \\
I_{\mathrm{v}-} \\
I_{\mathrm{Z}+} \\
I_{\mathrm{Z}-} \\
V_{\mathrm{W}+} \\
V_{\mathrm{W}-}
\end{array}\right)=\left(\begin{array}{cccc}
0 & 0 & 0 & 0 \\
0 & 0 & 0 & 0 \\
g_{\mathrm{m}} & -g_{\mathrm{m}} & 0 & 0 \\
-g_{\mathrm{m}} & g_{\mathrm{m}} & 0 & 0 \\
0 & 0 & \alpha_{\mathrm{w}+} & 0 \\
0 & 0 & 0 & \alpha_{\mathrm{w}-}
\end{array}\right)\left(\begin{array}{l}
V_{\mathrm{v}+} \\
V_{\mathrm{v}-} \\
V_{\mathrm{Z}+} \\
V_{\mathrm{Z}-}
\end{array}\right)
$$

where $g_{\mathrm{m}} \cong 1 / R_{\mathrm{M}}, \alpha_{\mathrm{w}_{+}}=1-\varepsilon \mathrm{v}_{+}$and $\alpha_{\mathrm{w}-}=1-\varepsilon_{\mathrm{v}-}$. Here, $\varepsilon_{\mathrm{v}+}$ and $\left.\varepsilon_{\mathrm{v}-}\left(\left|\varepsilon_{\mathrm{v}+}\right|,\left|\varepsilon_{\mathrm{v}-}\right| \ll\right) 1\right)$ represent voltage tracking errors of FB-VDBA. Also, $\alpha_{\mathrm{w}+}$ and $\alpha_{\mathrm{w}-}$ are ideally equal to unity. It should be noted that FB-VDBA has a pair of high-impedance voltage inputs $V_{\mathrm{v}+}$ and $V_{\mathrm{v}-}$, a pair of high-impedance current outputs $Z_{+}, Z_{-}$ and low-impedance voltage outputs $V_{\mathrm{W}+}$ and $V_{\mathrm{W}-}$.

FB-VDBA implementation by two integrated circuits OPA860 is shown in Fig. 2 [6]. OPA860 contains the socalled diamond transistor and voltage buffer. The terminals of diamond transistor are namely $E$ (emitter), $B$ (base) and $C$ (collector) with intrinsic resistance $R_{\mathrm{SE}}$ of

\footnotetext{
* Department of Electrical and Electronics Engineering, Istanbul University, 34320, Istanbul, Turkey, ayesila@gmail.com
} 


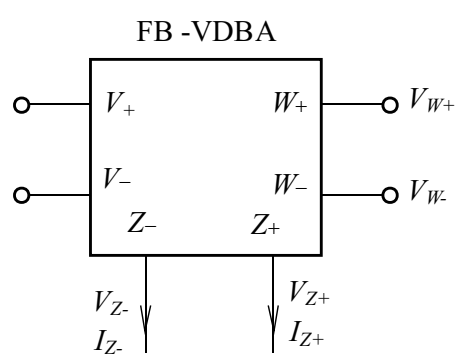

Fig. 1. The circuit symbol of the FB-VDBA

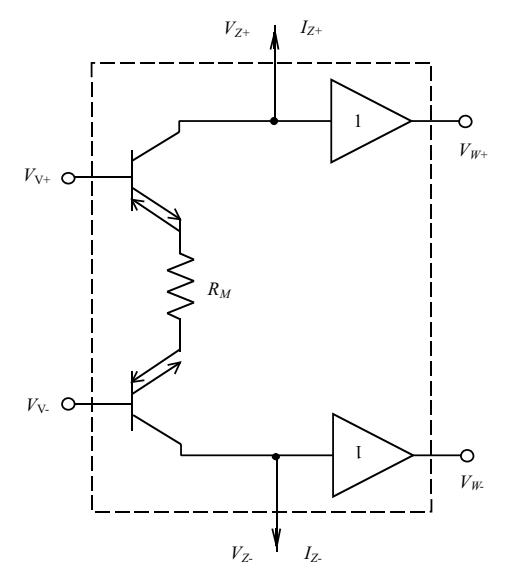

Fig. 2. Realization of FB-VDBA with OPA860 [6]

the $E$ terminal. In order to increase the linearity of collector current versus input voltage, a degenerating resistor $R_{\mathrm{M}} \gg R_{\mathrm{SE}}$ is added in series to the emitter [18]. The emitter terminals of diamond transistors in Fig. 2 are connected such that it forms a differential-input differentialoutput OTA.

\section{PROPOSED QUADRATURE OSCILLATOR CIRCUIT}

Figure 3 shows the circuit diagram of the proposed voltage-mode quadrature sinusoidal oscillator that employs a single FB-VDBA and three external passive components (one resistor and two capacitors). The proposed circuit has two voltage outputs. The presented topology is simple and employs all-grounded capacitors, which is more preferable to floating components because of the ease of integrated circuit implementation.

The assumption of an ideal FB-VDBA is characterized by (1), and the performance of a routine circuit analysis yields the characteristic equation expressed in equation

$$
s^{2}+s \frac{R_{\mathrm{M}}-R_{1}}{C_{1} R_{1} R_{\mathrm{M}}}+\frac{1}{C_{1} C_{2} R_{1} R_{\mathrm{M}}}=0 .
$$

Using (2), the proposed circuit can produce oscillation if

$$
R_{1} \geq R_{\mathrm{M}}
$$

The oscillation frequency of the oscillator is determined by

$$
f_{0}=\frac{1}{2 \pi} \sqrt{\frac{1}{C_{1} C_{2} R_{1} R_{\mathrm{M}}}} .
$$

The relation between the two output voltages at the oscillation frequency is

$$
V_{O 1}=e^{-j 90} k V_{O 2} . \quad k=\sqrt{\frac{C_{1} R_{1}}{C_{2} R_{\mathrm{M}}}} .
$$

Ensuring that the voltages $V_{O 1}$ and $V_{O 2}$ are quadrature. It is seen from (5) that if $k=1$ is chosen, the output voltage amplitudes equal to each other.

\section{EFFECT OF NON-IDEALITIES AND PARASITIC ELEMENTS}

The main parasitic elements which belong to the proposed oscillator circuit in Fig. 3 are shown in Fig. 4. The port $V+, V-, Z+$ and $Z-$ exhibit of high value parasitic resistance $R_{\mathrm{PJ}}$, in parallel with a low value capacitance $C_{\mathrm{PJ}}$ ( $j$ belongs to corresponding terminal names of FB-VDBA, which is $V+, V-, Z+$ and $Z-$ ) in Fig. 4. In the presence of these parasitic elements, the circuit given in Fig. 3 can be modified to Fig. 4 where $C_{\mathrm{P} 1}=C_{\mathrm{PV}-}+C_{\mathrm{PZ}-}, C_{\mathrm{P} 2}=C_{\mathrm{PZ}+}, R_{\mathrm{P} 1}=R_{\mathrm{PV}-} / R_{\mathrm{PZ}-}$, $R_{\mathrm{P} 2}=R_{\mathrm{PZ}+}$ and $R_{\mathrm{M}}^{\prime}=R_{\mathrm{M}}+R_{\mathrm{SE}}, R_{\mathrm{SE}}$ is the emitter resistance of diamond transistor. All parasitic elements except $R_{\mathrm{SE}}$ appear between respects to terminals and ground. It should be pointed out that the parasitic capacitances $C_{\mathrm{P} 1}$ and $C_{\mathrm{P} 2}$ can be absorbed into the external capacitances $C_{1}$ and $C_{2}$ as they appear in shunt with them.

Taking into consideration the non-idealities and parasitic elements of the FB-VDBA, the characteristic equation gets modified and can also be expressed,

$$
\begin{array}{r}
s^{2}+s\left(\frac{1}{C_{\mathrm{T} 2} R_{\mathrm{P} 2}}+\frac{1}{C_{\mathrm{T} 1} R_{\mathrm{P} 1}}-\frac{1}{C_{\mathrm{T} 1} R_{\mathrm{M}}^{\prime}}+\frac{1}{C_{\mathrm{T} 1} R_{1}}\right)+ \\
\frac{R_{\mathrm{M}}^{\prime}\left(R_{1}+R_{\mathrm{P} 1}\right)-R_{1} R_{\mathrm{P} 1}}{C_{\mathrm{T} 1} C_{\mathrm{T} 2} R_{1} R_{\mathrm{M}}^{\prime} R_{\mathrm{P} 1} R_{\mathrm{P} 2}}+\frac{\alpha_{\mathrm{WP}}}{C_{\mathrm{T} 1} C_{\mathrm{T} 2} R_{1} R_{\mathrm{M}}^{\prime}}=0
\end{array}
$$

where $C_{\mathrm{T} 1}=C_{1}+C_{\mathrm{P} 1}$ and $C_{\mathrm{T} 2}=C_{2}+C_{\mathrm{P} 2}$. The modified oscillation frequency and oscillation condition can be obtained as

$$
\begin{gathered}
f_{0 \mathrm{P}}=\frac{1}{2 \pi} \sqrt{\frac{R_{\mathrm{M}}^{\prime}\left(R_{1}+R_{\mathrm{P} 1}\right)-R_{1} R_{\mathrm{P} 1}}{C_{\mathrm{T} 1} C_{\mathrm{T} 2} R_{1} R_{\mathrm{M}}^{\prime} R_{\mathrm{P} 1} R_{\mathrm{P} 2}}+\frac{\alpha_{\mathrm{WP}}}{C_{\mathrm{T} 1} C_{\mathrm{T} 2} R_{1} R_{\mathrm{M}}^{\prime}}}, \\
R_{1} \geq R_{\mathrm{M}}^{\prime} /\left(1-\frac{C_{\mathrm{T} 1} R_{\mathrm{M}}^{\prime}}{C_{\mathrm{T} 2} R_{\mathrm{P} 2}}-\frac{R_{\mathrm{M}}^{\prime}}{R_{\mathrm{P} 1}}\right)
\end{gathered}
$$

It is seen from (7) and (8) that the oscillation frequency and oscillation condition are slightly affected by non-ideal and parasitic effects. From Fig. 4, the modified voltage transfer function from $V_{01}$ to $V_{02}$ 
$R_{1}$

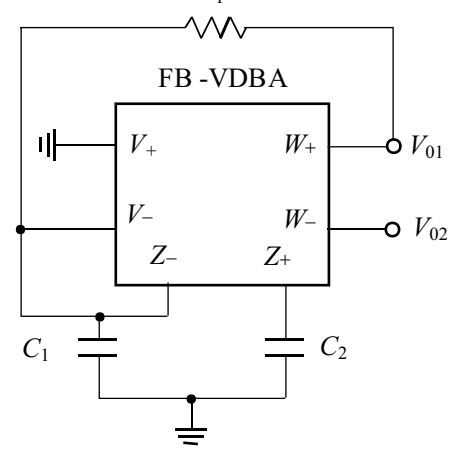

Fig. 3. Proposed quadrature oscillator circuit

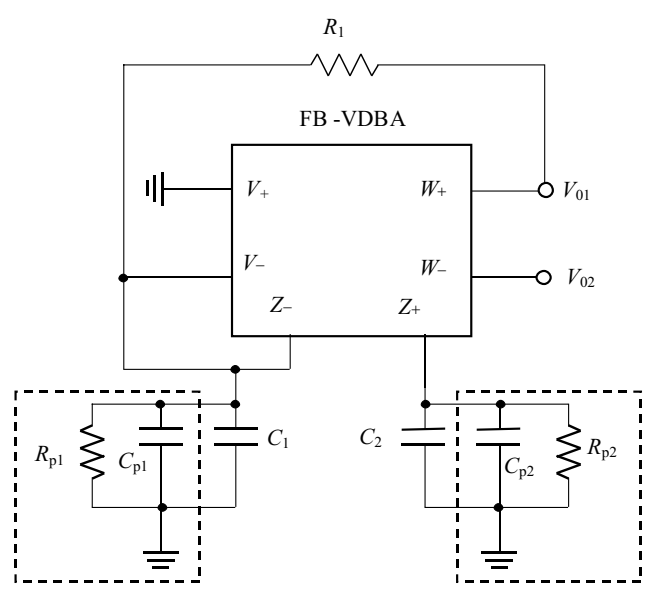

Fig. 4. The proposed oscillator including the parasitic elements

$$
\begin{gathered}
V_{01}=e^{-j \Phi} k_{\mathrm{P}} V_{02} ; \quad k_{\mathrm{P}}=\left\{\left[\alpha_{\mathrm{W}+}^{2} C_{\mathrm{T} 1} R_{1} R_{\mathrm{P} 1} R_{\mathrm{P} 2}^{2}\right] /\right. \\
{\left[\alpha _ { \mathrm { W } - } ^ { 2 } R _ { \mathrm { M } } ^ { \prime } \left[C_{\mathrm{T} 1} R_{1} R_{\mathrm{M}}^{\prime} R_{\mathrm{P} 1}+C_{\mathrm{T} 2} R_{\mathrm{P} 2}\left(R_{1}\left(R_{\mathrm{M}}^{\prime}-R_{\mathrm{P} 1}\right)\right.\right.\right.} \\
\left.\left.\left.\left.+R_{\mathrm{P} 1}\left(R_{0}^{\prime}+\alpha_{\mathrm{W}+} R_{\mathrm{P} 2}\right)\right)\right]\right]\right\}^{1 / 2}
\end{gathered}
$$

The phase difference, $\Phi_{\mathrm{P}}$, between $V_{01}$ and $V_{02}$ is

$$
\begin{aligned}
\Phi_{\mathrm{P}}= & \tan ^{-1}\left(C_{\mathrm{T} 1} R_{1} R_{\mathrm{M}}^{\prime} R_{\mathrm{P} 1} / C_{\mathrm{T} 2} R_{\mathrm{P} 2}\left(R_{1} R_{\mathrm{M}}^{\prime}-\right.\right. \\
& \left.\left.R_{1} R_{\mathrm{P} 1}+R_{\mathrm{M}}^{\prime} R_{\mathrm{P} 1}+R_{\mathrm{P} 1} R_{\mathrm{P} 2} \alpha_{\mathrm{W}+}\right)\right)^{1 / 2}-90 .
\end{aligned}
$$

The values of parasitic capacitances and resistors taken from the OPA860 (datasheet-OPA860) are $C_{\mathrm{PV}-}=$ $2.1 \mathrm{pF}, C_{\mathrm{PZ}-}=4.1 \mathrm{pF}, C_{\mathrm{PZ}+}=4.1 \mathrm{pF}, R_{\mathrm{SE}}=21 \Omega$, $R_{\mathrm{PV}-}=455 \mathrm{k} \Omega, R_{\mathrm{PZ}-}=R_{\mathrm{PZ}+}=51.23 \mathrm{k} \Omega$ and $\alpha_{\mathrm{W}+}=$ $\alpha_{\mathrm{W}-}=0.98$. Using given the other values in detail in the next section, $f_{0}=1.284 \mathrm{MHz}, k=1.024, \Phi=-90^{\circ}$, $f_{0 \mathrm{P}}=1.264 \mathrm{MHz}, k_{\mathrm{P}}=1.036$ and $\Phi_{\mathrm{P}}=-89.86^{\circ}$ are found as numerical. From (7),(9) and (10) it is obvious that the modified oscillation frequency, voltage amplitudes and phase difference are slightly altered due to the non-ideal gains and parasitic effects of the FB-VDBA. However, these differences can be fixed with values of internal resistors.

\section{EXPERIMENTAL EVALUATION}

Designed oscillator was realized with surface-mounted (SMD) 1206 sized passive elements and SOIC packaged OPA860 integrated circuits. Resistors $R_{3}$ and $R_{4}$ have a value of $330 \Omega$ which provide diamond transistor DC biasing. For each OPA860, a resistor of $100 \Omega$ in series with the base of OTA is connected as recommended in datasheet [18]. $C_{1}$ and $C_{2}$ are capacitors that are required for oscillation, $C_{3}$ and $C_{4}$ are bypassing capacitors. Here, all of the capacitors are multilayer ceramic capacitors and the tolerances of these capacitors are much larger than the input capacitances. $R_{\mathrm{M}}$ is taken $100 \Omega$ and $R_{1}$ consists of serially connected resistors $100 \Omega$ and $27 \Omega$, so that, the oscillation condition $R_{1}>R_{\mathrm{M}}$ is achieved. Additionally, a temperature sensor IC is mounted on PCB for measurement of ambient temperature. Circuit is powered with adjustable output dual power supply (Instek, GPC-3060D).

With $\pm 5 \mathrm{~V}$ power supply and $47 \Omega$ resistive loads with $C_{1}=C_{2}=1 \mathrm{nF}$, signals viewed at the outputs of the oscillator are given in Fig. 5(a). Frequency of the oscillator is measured as $1.333 \mathrm{MHz}$ and phase difference between outputs is $90^{\circ}$, where ideal oscillation frequency is theoretically found as $1.284 \mathrm{MHz}$, respectively. The error of the oscillation frequency between theoretically and experimental results is to be $3.8 \%$. The discrepancy between ideal and measured oscillation frequency mainly stems from actual values of the resistors and capacitors and the parasitic impedances of FB-VDBA. Taking into account parasitic elements in (7), oscillation frequency is calculated as $1.264 \mathrm{MHz}$. Current consumption is $80 \mathrm{~mA}$. To see amplitude variation with the time and phase difference between two outputs more clearly, Lissajous curves are viewed, Fig. 5(b). The circle that has a constant radius indicates constant amplitude and $90^{\circ}$ phase difference. Frequency spectrum of each signal output is displayed as given in Fig. 6. To obtain a lower oscillation frequency, $C_{1}$ and $C_{2}$ capacitors are replaced with $10 \mathrm{nF}$, respectively. For this condition, calculated oscillation frequency value is $128.4 \mathrm{kHz}$, and measured value is $122 \mathrm{kHz}$.

Total Harmonic Distortion (THD) value and amplitude of output signals are measured for different power supply levels and two different oscillation frequencies (Table-1). As supply level increases, THD generally increases. When supply is $\pm 4 \mathrm{~V}$ and oscillation frequency is $1.33 \mathrm{MHz}$, THD measured as $0.51 \%$ and $0.98 \%$ at $V_{01}$ and $V_{02}$ outputs, respectively. However, with same power supply level, THD values of output signals are increased to $1.12 \%$ and $1.20 \%$ when oscillation frequency is decreased to $122 \mathrm{kHz}$. At $1.33 \mathrm{MHz}, V_{01}$ output always has a lower THD compared to $V_{02}$ output for all supply levels. At $122 \mathrm{kHz}, V_{01}$ output has higher THD compared to $V_{02}$ output when only $\left|V_{\mathrm{CC}}\right|>5.5 \mathrm{~V}$ and $\left|V_{\mathrm{EE}}\right|>5.5 \mathrm{~V}$. For all supply levels and oscillation frequencies, amplitude of 


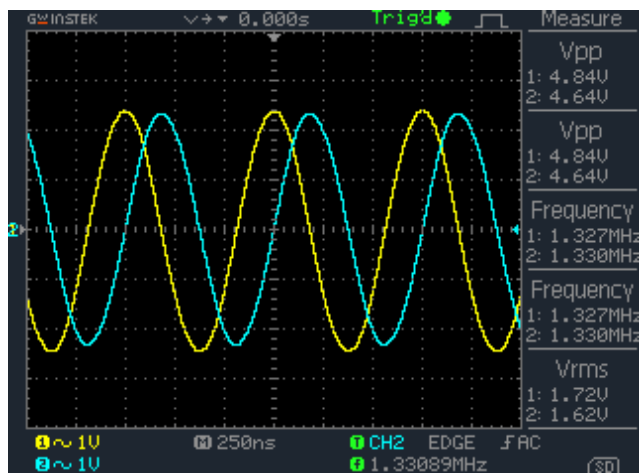

(a)

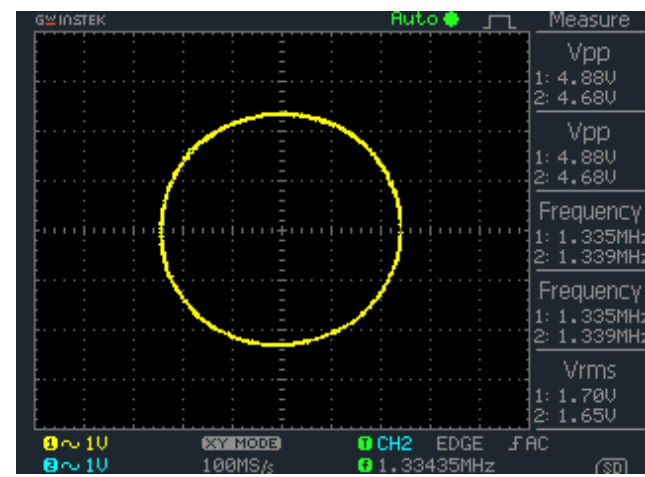

(b)

Fig. 5. Output signals displayed at oscilloscope in (a) - normal mode and (b) $-X / Y$ mode

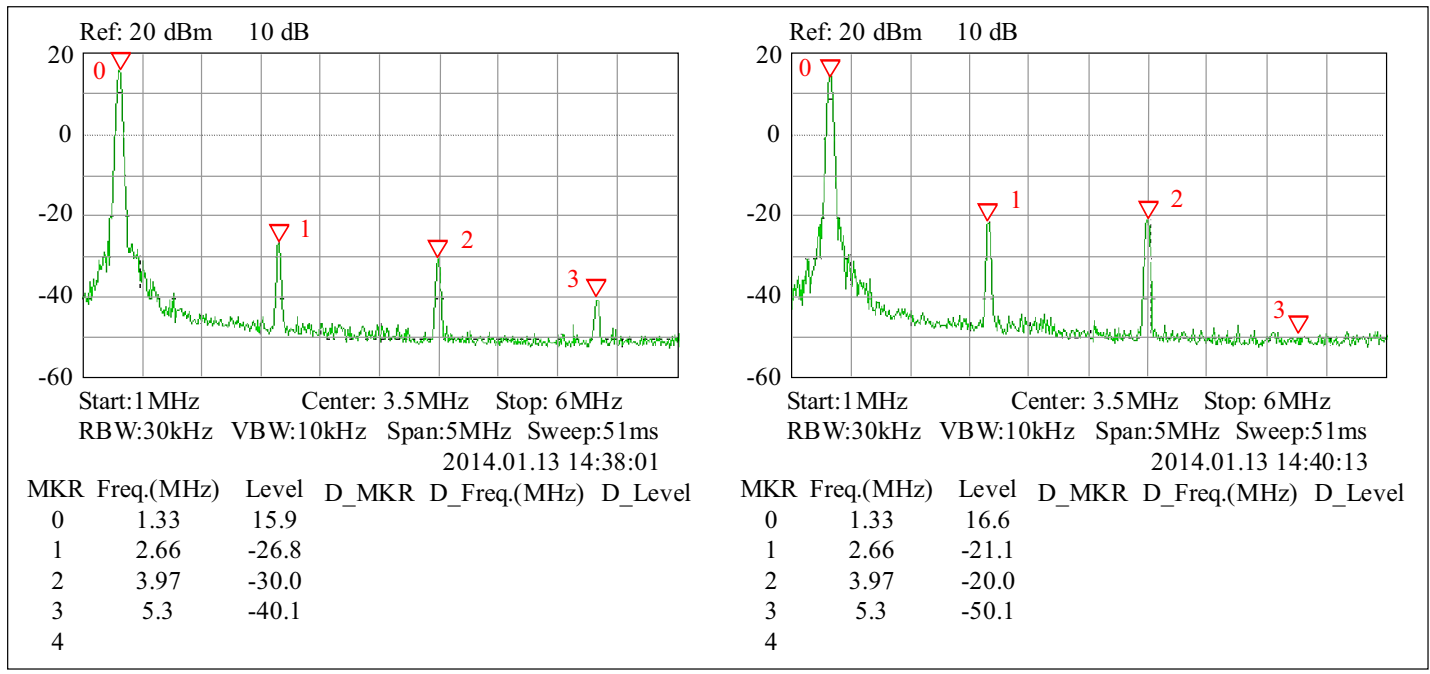

Fig. 6. Frequency spectrum at (left) $-V_{O 1}$ and (right) $-V_{O 2}$ output of the oscillator

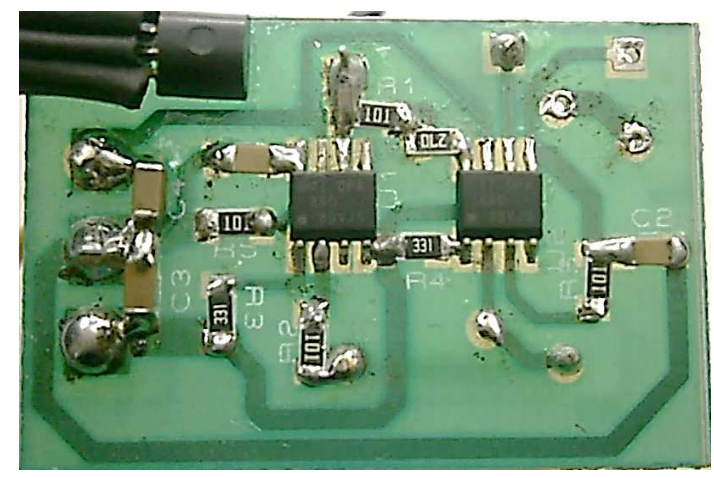

(a)

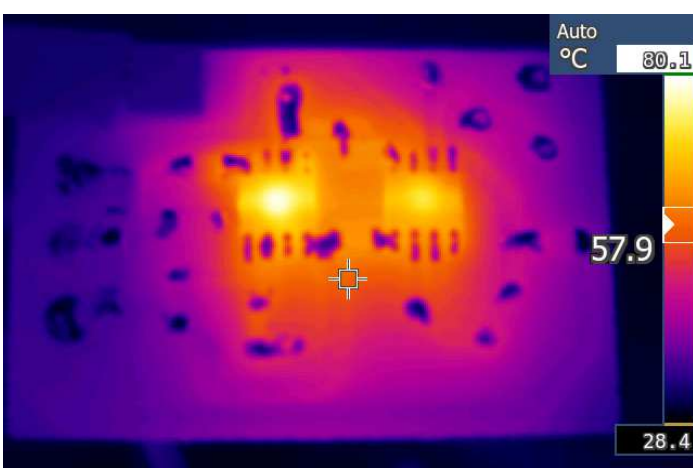

(b)

Fig. 7. PCB photograph of the realized oscillator and thermal image of the oscillator after 48 hours of operation

Table 1. Effect of supply level to output THD and amplitude $@ 27^{\circ} \mathrm{C}$

\begin{tabular}{|c|c|c|c|c|c|c|c|c|c|}
\hline \multirow{2}{*}{\multicolumn{2}{|c|}{ Supply (V) }} & \multicolumn{8}{|c|}{$C_{1}=C_{2}=10 \mathrm{nF}\left(f_{0}=122 \mathrm{kHz}\right) C_{1}=C_{2}=1 \mathrm{nF}\left(f_{0}=133 \mathrm{MHz}\right)$} \\
\hline & & \multicolumn{2}{|c|}{$\begin{array}{c}\text { Amplitudes } \\
\left(\mathrm{V}_{\mathrm{p}-\mathrm{p}}\right)\end{array}$} & \multicolumn{2}{|c|}{$\begin{array}{c}\text { THD } \\
(\%)\end{array}$} & \multicolumn{2}{|c|}{$\begin{array}{c}\text { Amplitudes } \\
\left(\mathrm{V}_{\mathrm{p}-\mathrm{p}}\right)\end{array}$} & \multicolumn{2}{|c|}{$\begin{array}{c}\text { THD } \\
(\%)\end{array}$} \\
\hline $\mathrm{V}_{\mathrm{CC}}$ & $\mathrm{V}_{\mathrm{EE}}$ & $\mathrm{V}_{01}$ & $\mathrm{~V}_{02}$ & $\mathrm{~V}_{01}$ & $\mathrm{~V}_{02}$ & $\mathrm{~V}_{01}$ & $\mathrm{~V}_{02}$ & $\mathrm{~V}_{01}$ & $\mathrm{~V}_{02}$ \\
\hline 6.0 & -6.0 & 6.04 & 5.64 & 5.56 & 4.42 & 5.80 & 5.48 & 1.23 & 3.24 \\
\hline 5. & & & 5.2 & & & 5.36 & & & \\
\hline 5.5 & -4.5 & 4.84 & 4.68 & 1.89 & 1.9 & 4.72 & 4.52 & 0.89 & 1.70 \\
\hline 5.0 & -5.0 & 5.08 & 4.7 & 2.13 & & 4.88 & & 0.91 & \\
\hline & -5.5 & 4.8 & 4.6 & 1.6 & & 4.7 & 4. & 1.0 & \\
\hline 4.5 & -4.5 & 4.44 & 4.24 & 1.41 & 1.59 & 4.32 & 4.16 & 0.82 & 1.63 \\
\hline 4.0 & -4.0 & 3.92 & 3.76 & 1.12 & 1.20 & 3.72 & 3.60 & 0.51 & 0.98 \\
\hline
\end{tabular}




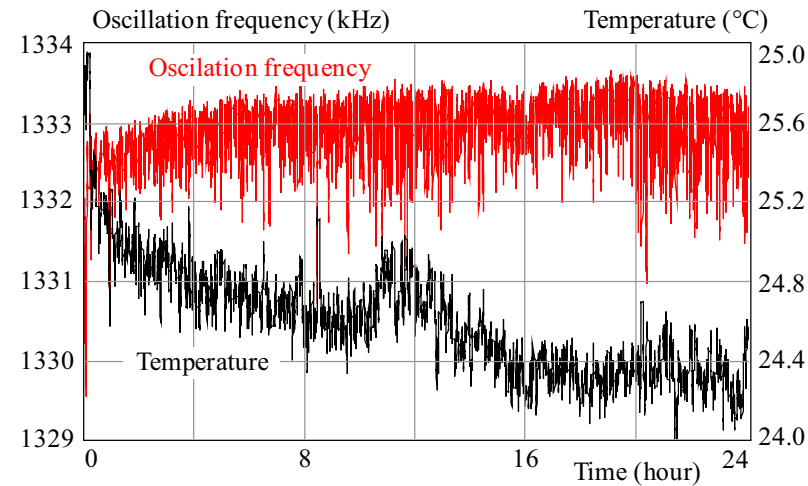

Fig. 8. 24-hour graph of oscillation frequency and ambient temperature

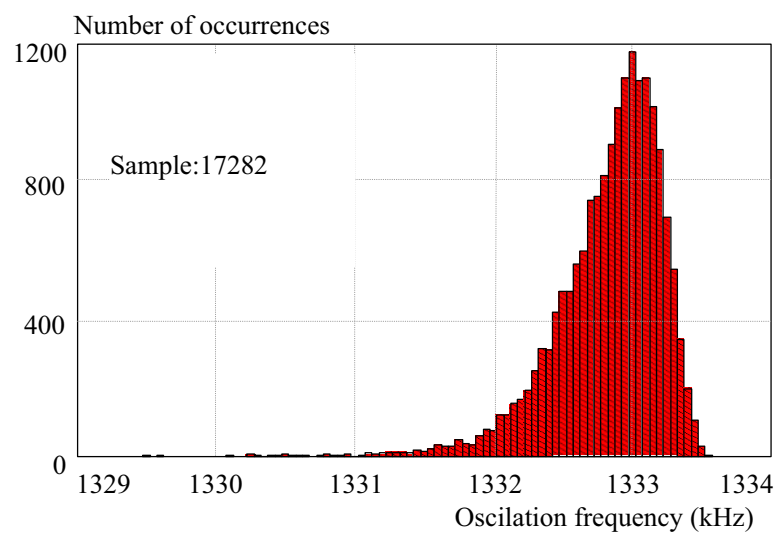

Fig. 9. Histogram of recorded oscillation frequency data

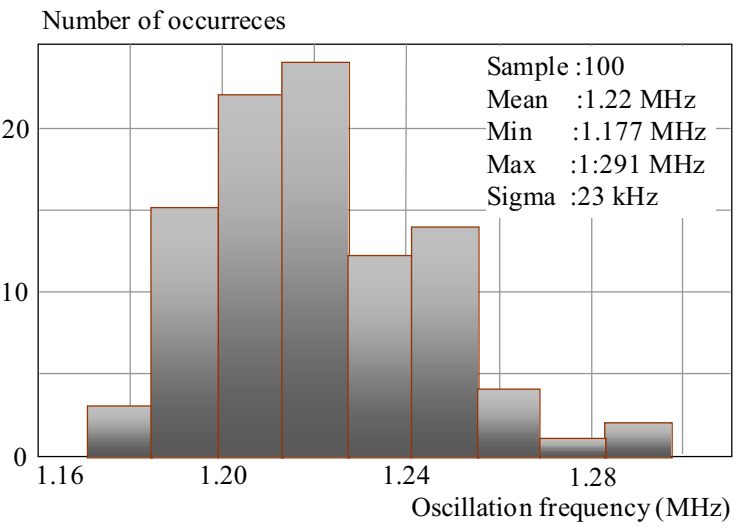

Fig. 10. Histogram of the oscillation frequency distribution

$V_{01}$ output is always higher then $V_{02}$ output. Equation (5) yields the nearly constant ratio of amplitudes.

In Fig. 7, the layout of realized oscillator and the printed circuit board (PCB) thermal image are shown revealing that the two OPA860 integrated circuits have different temperatures, so, internal resistances of diamond transistors are different and this difference has to be considered in the calculation of the oscillation frequency.

To determine deviation of the frequency and continuity of oscillation, a long time data recording has been made. To measure temperature of environment, temperature sensor IC that has been stick at the top of PCB is used. Voltage output of the sensor and frequency of the oscillator measured at the same time with 5.5 digit precision multi-meters (Agilent 34405, 34450A) and measured data recorded with Agilent IO software. As given in Fig. 8, during a 24 hours of operating time, oscillation frequency is constant with respect to the decreasing ambient temperature. In Fig. 9, the histogram of the recorded frequency data is given. By taking nominal frequency as $1.333 \mathrm{MHz}$, the oscillation frequency has a deviation of $+0.5 /-4 \mathrm{kHz}$ and no damping occurred during the 24 hours operation.

To investigate the influence of passive components tolerances on the oscillation frequency, Monte Carlo analysis was performed for 100 samples in shown Fig. 10 selecting as $C_{1}=C_{2}=1 \mathrm{nF}, R_{\mathrm{M}}=100 \Omega$ and $R_{1}=127 \Omega$. Tolerances of passive components were considered $1 \%$ for SMD resistors and $10 \%$ for capacitors. The oscillation frequency of the proposed oscillator shift has a minimum value of $1.177 \mathrm{MHz}$ and maximum value of $1.291 \mathrm{MHz}$.

\section{CONCLUSION}

This paper presents an attractive and new quadrature oscillator with minimum number of active and passive elements. The proposed oscillator is based on FB-VDBA that offers two voltage outputs. This paper describes the related governing equations of the proposed oscillators, and presents a discussion of the non-ideality analysis and parasitic effect of the circuits. The effectiveness of this circuit was verified by the experimental results, which show good agreement with the theoretical analysis. FBVDBA can be easily realized by using a commercially available active device such as an OPA860. Therefore, the proposed oscillator circuit can be produced commercially. Because of their simplicity and versatility, the proposed oscillator provides new possibilities for the application of FB-VDBA device; the oscillators offer potential for wide application in instrumentation, measurement, and electronic systems.

\section{REFERENCES}

[1] HOROWITZ, P.-HILL, W.: The Art of Electronics, Cambridge University Press, Cambridge, U.K., 1991.

[2] TIETZE, U.-SCHENK, C.: Electronic Circuits: Design and Applications, Springer, Berlin, Germany, 1991.

[3] HOLZEL, R.: A Simple Wide-Band Sine Wave Quadrature Oscillator, IEEE Trans. Instrum. Meas. 42 (1993), 758-760.

[4] KACAR, F.-YESIL, A.-NOORI, A. : New CMOS Realization of Voltage Differencing Buffered Amplifier and its Biquad Filter Applications, Radioengineering 21 No. 1 (2012), 333-339.

[5] PRASAD, D.-BHASKAR, D. R.-PUSHKAR, K. L. : Realization of New Electronically Controllable Grounded and Floating Simulated Inductance Circuits using Voltage Differencing Differential Input Buffered Amplifiers, Active and Passive Electronic Components (2011). 
[6] BIOLKOVA, V.-KOLKA, Z.-BIOLEK, D. : Fully Balanced Voltage Differencing Buffered Amplifier and its Applications, Proc. of the 52nd MWSCAS, 2009, pp. 45-48.

[7] BIOLEK, D.-BIOLKOVA, V.-KOLKA, Z.: All-Pass Filter Employing Fully Balanced Voltage Differencing Buffered Amplifier, Proc. of IEEE Latin American Symposium on Circuits and Systems, 2010, pp. 232-235.

[8] SOTNER, R.-JERABEK, J.-HERENCSAR, N.: Voltage differencing Buffered/Inverted Amplifiers and their Applications for Signal Generation, Radioengineering 22 No. 2 (2013), 490-504.

[9] WANG, C.-LIN, H. : Multifunction Current Differencing Cascaded Transconductance Amplifier (MCDCTA) and Its Application to Current-Mode Multiphase Sinusoidal Oscillator, Journal of Electrical Engineering 66 No. 5 (2015), 241-249.

[10] LAHIRI, A.: Novel Voltage/Current-Mode Quadrature Oscillator using Current Differencing Trans-Conductance Amplifier, Analog Integr. Circ. Sig. Process. 61 (2009), 199-203.

[11] HORNG, J. W. : Quadrature Oscillators using Operational Amplifiers, Active and Passive Electronic Components (2011), Article ID 320367.

[12] TANGSRIRAT, W.-PRASERTSOM, D.-PIYATAT, T.SURAKAMPONTORN, W : Single-Resistance-Controlled Quadrature Oscillator using Current Differencing Buffered Amplifiers, International Journal of Electronics 95 No. 11 (2008), $1119-1126$

[13] TANGSRIRAT, W.-SURAKAMPONTORN, W.: Single-Resistance-Controlled Quadrature Oscillator and Universal Biquad Filter using CFOAs, Int. J. Electron. Commun. (AEÜ) 63 (2009), 1080-1086.

[14] KUMNGERN, M.-DEJHAN, K.: DDCC-Based Quadrature Oscillator with Grounded Capacitors and Resistors, Active and Passive Electronic Components (2009), Article ID987304.

[15] HERENCSR, N.—VRBA, K.-KOTON, J.—LAHIRI, A. : Realizations of Single-Resistance-Controlled Quadrature Oscillators using a Generalized Current Follower Trans-Conductance Amplifier and a Unity-Gain Voltage-Follower, International Journal of Electronics 97 No. 8 (2010), 897-906.

[16] HORNG, J. W.-HOU, C. L.-CHANG, C. M.-CHOU, H. P.-LIN, C. T.-WEN, Y. H.: Quadrature Oscillators with Grounded Capacitors and Resistors Using FDCCIIs, ETRI Journal 28 No. 4 (2006), 486-494.
[17] SOTNER, R.-HERENCSAR, N.-JERABEK, J.-DVORAK, R.-KARTCI, A.-DOSTAL, T.-VRBA, K.: New Double Current Controlled CFA (DCC-CFA) Based Voltage-Mode Oscillator with Independent Electronic Control of Oscillation Condition and Frequency, Journal of Electrical Engineering 64 No. 2 (2013), 65-75.

[18] OPA860 - Wide Bandwidth Operational Trans-conductance Amplifier (OTA) and Buffer. Texas Instruments, SBOS331C June 2005 - Revised August 2008, www.ti.com.

Received 6 October 2015

Abdullah Yesil received BSc, MSc and $\mathrm{PhD}$ degrees from Istanbul University, both in electrical and electronics engineering in 2009, 2011 and 2016, respectively. Since 2012, he has been working as a research and teaching assistant in Istanbul University. His main research interests are active network synthesis, current-mode circuit design, low-noise implementation of continuous time filters, MOS-only filter design and electronic circuits for computer aided design.

Firat Kacar received his BSc, MSc and $\mathrm{PhD}$ degrees from Istanbul University, all in electrical and electronics engineering 1998, 2001 and 2005. He is currently an associate professor at the Electrical and Electronics Engineering Department of Istanbul University. His current research interests include analog circuits, active filters, synthetic inductors, CMOS based circuits electronic device modeling and hot-carrier effect on MOS transistor. He is the author or co-author of about 70 papers published in scientific journals or conference proceedings.

Koray Gurkan received the $\mathrm{MSc}$ and $\mathrm{PhD}$ degree in biomedical engineering from Istanbul University, Istanbul, in 2006 and 2012, respectively. Since 2005, he has been working as a research assistant at Istanbul University, Department of Electrical and Electronics Engineering. His current research interests include metrology, sensor interface, micro-controller based circuit design. He also carried out investigations about telemetric measurements, electronic systems of electric, hydrogen and solar cars. 